\title{
Innovative Technology for Sustainable New Materials
}

\author{
V. Beghetto ${ }^{* 1,2}$ L. Agostinis ${ }^{1}$, R. Taffarello ${ }^{2}$, R. Samiolo ${ }^{2}$
}

\begin{abstract}
:
Active Cross-Linking agents (ACL) are a class of compounds used as activators for a wide range of reactions which aim to make covalent bonds without leaving trace in the final product. Crossing S.r.l., an Innovative Start Up, made a study to synthesize and test a new type of ACL for different manufacturing processes. In particular, a process was developed for the tanning of hides, allowing the making of a stable and metal-free leather with a deep focus to the environmental sustainability. Furthermore a second process for the production of active packaging by ACL is reported preventing the alteration of food, thanks to the complete, or partial, removal of preservatives and allowing a concrete solution to allergenic and intolerance problems. With this study Crossing S.r.l wants to demonstrate the possibility to produce good quality leather or offering an alternative to the use of preservatives with a highly sustainable protocols.
\end{abstract}

Keywords: Cross-Linking, Innovative, Sustainable, Industry, packaging, cellulose, leather.

\section{Introduction}

Consumers living in industrialized countries pay much attention to health and ecological issues. No records have been made, but if at least $2 \%$ of EU citizens can be reached, over 10 million potential customers are available. The EU Commission is working to achieve a circular economy requiring to create highly durable goods, high performance, efficient and environmental friendly processes, with reduction of hazardous materials and of waste production.

Crossing S.r.l., an Academic Spin-Off and Innovative Start Up, is developing new environmental technologies based on the use of Cross-Linking Agents (ACL).

ACL technology is designed to meet requirements set by the European commission in terms of environmental sustainability and production.

ACL are activating agents for condensation, cross-linking or grafting reactions, capable of forming covalent amide, ester, and thioester bonds by reaction between carboxylic groups and amine, alcohols or tioalcohols.

Condensation reactions are the basis for the synthesis of all the important biological macromolecules (carbohydrates, proteins, lipids, nucleic acids) from their simpler subunits. Condensation is not spontaneous and thus it require to be activated; this can be done by heating (at $\mathrm{T} 160-180^{\circ} \mathrm{C}$ ) [1], or in milder reaction conditions (eg. $\mathrm{T} \mathrm{ca} .25-50^{\circ} \mathrm{C}$ ) in the presence of a chemical "activating" reagent [2,3]. Carbodiimides are commonly used for these reaction since in the presence of a carboxylic acid, an intermediate active species is formed, which in the presence of an amine, alcohol or thiol allows to obtain

$\mid{ }^{1}$ Department of Molecular Sciences and Nanosystems, University Ca' Foscari of Venice, Via Torino 155, 30172 Venice, ITALY.beghetto@unive.it, lodovico.agostinis@hotmail.com

${ }^{2}$ Crossing S.r.l., Piazza delle Istituzioni n. 27 ed. H, 31100 Treviso, ITALY. renzotaffarello@hotmail.com, riccardosamiolo@libero.it 
the corresponding amide, ester or thioester [4]. Dicyclohexylcarbodiimide (DCC) is among the most commonly used carbodiimides; however, DCC requires the use of organic solvents and leads to the formation of a toxic co-product that must be carefully removed at the end of the reaction.

Alternatively, 1-ethyl-3-(3-dimethylaminopropyl) carbodiimide hydrochloride (EDC) may be employed in water. However, to be efficient EDC requires the use of equimolar or greater amount of N-hydroxysuccinimide (NHS). Furthermore, EDC is not very stable, must be stored at low temperature (about $-20^{\circ} \mathrm{C}$ ) and is expensive. Despite these disadvantages, EDC remains one of the most frequently used reagents for the synthesis of polyaminoacids and other pharmaceutical derivatives with high added value, as well as for cross-linking of collagen, for the reconstruction of tendons, etc. [5,6,7].

In this work we wish to highlight some of the main achievements on the application of ACL reported by Crossing in these last two years.

\section{Results and discussion}

The great potential of ACL molecules is embedded in their multiple applications in different manufacturing realities. ACL differ from other active cross-linking agents in few main respects. In particular, in comparison to EDC:

-The reactivity in all cases tested of ACL is superior to EDC/NHS;

-EDC requires equimolar or higher amounts of NHS to implement its activity; ACL do not require any co reactant;

-The reactivity of EDC is highly dependent from the $\mathrm{pH}$ of the reaction, ACL can be used without $\mathrm{pH}$ control within a range of $4.5<\mathrm{pH}<10$;

-ACL may be recycled;

-ACL are easily removed after the reaction allowing easy purification of the final products. EDC/NHS usually requires further purification steps to remove completely the byproducts of the reaction formed.

The work carried out by Crossing has demonstrated that ACL are versatile activating agents which may carry out different types of reactions such as condensation, crosslinking, grafting.

\subsection{Cross-Linking}

Two highly significant examples of ACL as cross-linking agents are their application as tanning agents or for the production of antimicrobial modified cellulose films. A brief description of these two applications is given bellow:

\subsubsection{ACL as tanning agents}

Currently over $85 \%$ of the world's leather are tanned with chromium salts (III). The European production is responsible for $17 \%$ of the worldwide leather market. In 2013 according to UNIC (National Union of Tanning Industries), Italy has covered 62\% of the European production corresponding to $125.000 .000 \mathrm{~m}^{2}$ of leather each year with an annual turnover of 4.9 billion euro/year. Only in Italy, the total amount of chemical 
products used by the tanning industries is of ca. 47 million tons/year. [8]

The widespread use of chromium is due to the high quality of the leather produced, ease of use, availability, low cost, etc. However, it is known that chromium content in the skin can give rise to $\mathrm{Cr}$ (VI) species, highly toxic and carcinogenic. Currently, the main alternatives used industrially to tan leather (natural or synthetic tannins, aldehydes) impart physical and mechanical characteristics significantly lower than Chrome, without, however, solving the problem of health for the consumer and the environment impact since from this leather formaldehyde (a carcinogen) and phenol (cytotoxic) can be released. No real solution is known today and the quest for a substitution product is urgent.

Crossing, in its recent patent has reported that ACL are able to stabilize collagen and thus also hides without being "trapped" inside the collagen matrix, generating completely non-toxic and biocompatible leather providing a finished product with comparable characteristics to those achieved with chromium salts. Particularly relevant is the reduced environmental impact of these materials both for the consumer and also in terms of "end of life", since the morphological characteristics of the skin remain intact compared to those of the skin of the animal and the leather is totally free of heavy metals, formaldehyde, phenol, etc.

The fundamental and unprecedented difference between all conventional tanning agents and ACL is that the latter act as "catalysts" or "enzymes", are not retained in the leather after tanning. They stabilize the collagen structure, increase the thermal stability ( $\mathrm{Tg}$ ), and leave no trace giving tanned leather which is devoid chemicals and is thus non-toxic.

At present, it is commonly accepted that a tanning agent is such if during tanning it is retained within the collagen matrix. ACL refuted this definition showing that it is possible to tan hides without the need of any chemical remaining permanently bonded to the skin.

ACL overcome one of the most critical aspects of all alternative tanning systems known today which give leather of lower quality, applicability, price, etc. to chrome leather. ACL are the first real alternative to chrome salts verified to date, as will be further explained bellow. [9].

\subsubsection{ACL for active packaging:}

Many solutions exist today for preserving and extending food shelf's life such as plastic bags, trash can liners, plastic plates, etc. Nonetheless, in many cases preservatives are required to secure the quality of the goods.

A possible solution comes from active packaging defined as "packaging in which subsidiary constituents have been deliberately included in or on the packaging material to enhance the performance of the package system". In most cases active packaging is an additional part of the packaging and antimicrobial agents, if present, in most cases are added to the food with inevitable problems for the consumer.

ACL have been employed for the production of an innovative carboxymethylcellulose (CMC) film with the aim to produce an antimicrobial food packaging. In particular, CMC is frequently employed as starting material for the production of superabsorbent or gel $[10,11]$. CMC is inexpensive and is obtained by acetylation of cellulose; the degree of 
carboxylic groups present in CMC may vary from 0 to $100 \%$.

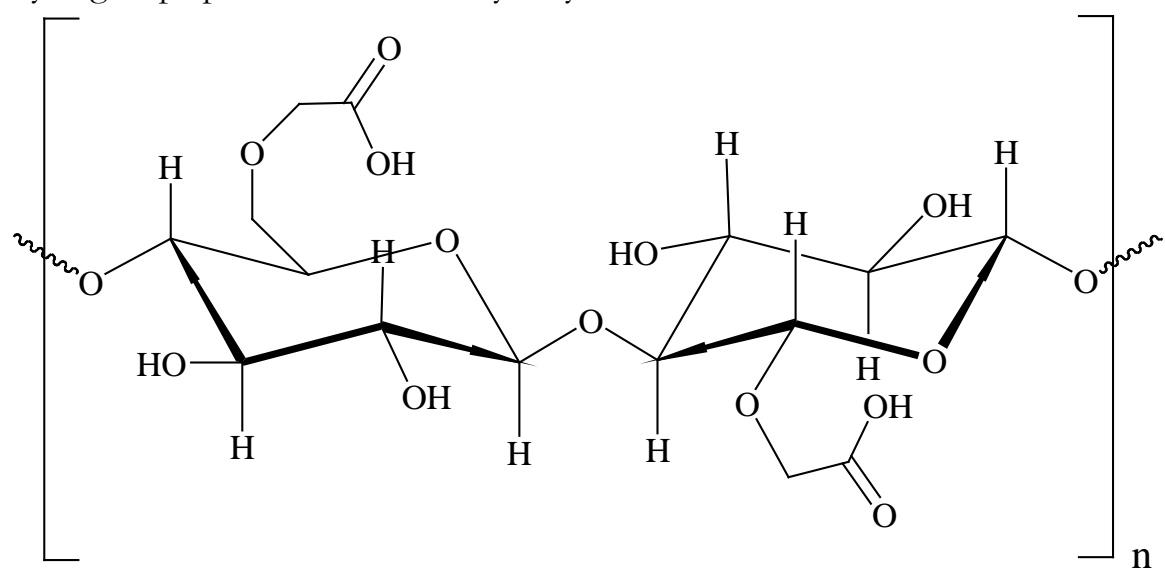

Figure 1. An example of CMC with 100\% degree of carboxylic groups.

We have carried out a set of experiments to verify the possibility of cross-linking CMC with ACL. From our data it has been highlighted that ACL are able to react the $-\mathrm{COOH}$ and - $\mathrm{OH}$ groups present in $\mathrm{CMC}$ between each other, and producing a film highly crosslinked. Moreover by adding an antimicrobial active agent, having functional groups able to react with CMC by way of ACL, an "active film" is obtained. Antimicrobial and preservation tests have shown that these films are highly active and may preserve food at room temperature, up to 20 days.

\subsection{Grafting}

As far as the field of synthetic materials is concerned, ACL are a useful tool for the production of grafted polymers or grafted surfaces (natural or synthetic). The grafting protocol requires a series of co-reagents, which may be used also in combination. In particular, Crossing has studied grafting reactions with the aim to produce antibacterial properties, anti-fouling, etc. polymers or surfaces. It is important to highlight that the activity of the co-grafted reagent should be preserved even in the final product so that the grafting is carried out in a conservative and not destructive manner.

\subsubsection{Polymer Graftin}

ACL have been employed for the synthesis of antimicrobial or antifouling polymers.

The antibacterial polymers currently present on the market can be divided into different classes according to the microbial action taking place and in particular: [12] I) polymers with intrinsic antimicrobial properties; II) polymers by chemical modifications of the structure; III) polymers with antibacterial molecules covalently bonded.

The latter is considered to be one of the most efficient ways to obtain polymers having specific activities, but it is currently very seldom employed due to synthetic requirements 
and high costs. ACL are a cost efficient and easy to use solution for covalently grafted antimicrobial or antifouling polymers.

Polyacrylic acid of different molecular weights have been tested as standard substrate for grafting experiments in the presence of ACL and a set of aromatic, aliphatic amines or alcohols selected according to their specific activities. Other possible functionalization by grafting can be achieved with quaternary ammonium salts derivatives[13].

\subsubsection{Surface Grafting}

ACL technology may be employed for the modification of synthetic polymeric surfaces in combination with plasma treatment. Polymeric surfaces made of PE, PP, PET, etc. are devoid of reactive functional groups; thus, pre-activation by plasma, corona or other modification techniques, is necessary to prepare the material for ACL treatment. Experiments are under way to verify this protocol for the preparation of antimicrobial surfaces.

\section{Conclusions}

In conclusion in this paper we have given a brief overview of some of the most relevant applications implemented by Crossing on the use of ACL for cross-linking, or grafting reactions.

In comparison to the state of the art, ACL technology is a very promising alternative to tanning, grafting and condensing agents, with significant advantages, and in particular:

\section{Tannery}

For the leather industry ACL compounds have been demonstrated to be efficient tanning agents able to tan hides:

- giving non-toxic final products of comparable quality to chrome tanned leather,

- no chrome is required for tanning. We estimate that if $10 \%$ of the European market where to adopt this technology 4000 ton/year of chrome will be substituted by ACL with a significant reduction in toxic waste disposal both liquid and solid;

- significant reduction in waste disposal costs;

-possibility for recovery of leather scraps as secondary raw material for high value added products.

\section{Active Packaging}

For the production of active antimicrobial or antifouling polymers (natural and synthetic) or surfaces, ACL technology may comply several advantages such as:

- the extension of the shelf life of fish, dairy, meat preparations, fruit and vegetables;

- specific treatments against pathogenic bacteria or responsible for the specific degradation for the conservation of food may be achieved;

- savings in the cost of additives compared to other employed today (for example silverbased ion);

- in the case of reusable packaging ACL technology is estimated to improve food security and reduce management costs. Moreover, active molecules are covalently bonded thus 
there is no release problems or migration of the antimicrobial/antifouling compounds inside the food.

\section{References}

B.S. Jursic and Z. Zdravkovski, 1993. A simple Preparation of Amides (III) from Acids (I) and Amines (II) by Heating of Their Mixture. Synth. Commun., 23, 2761-2770.

F. Albericio, R. Chinchilla, D.J.. Dodsworth and C. Najera, 2001. New trends in peptide coupling reagents. Organic preparations and procedures int., 33, 205-303.

I. Rault, V. Frei, D. Herbage, N. Adbul-Marak and A. Hue, 1996. Evaluation of different chemical methods for cros-linking collagen gel, films and sponges. J. Mater. Sci. Mater. Med., 7, 215-221.

A. El-Faham and F. Albericio, 2011. Peptide Coupling Reagents, More than a Letter Soup. Chem. Rev., 111, 6557-6602.

D.V. Shepherd, et al., 2015. The process of EDC-NHS Cross-linking of reconstituted collagen fibres increases collagen fibrillar order and alignment. APL Mater. , 3, 1-13;

G. Wnek, L. Meng, US9040665 B2.

L. Wenguang, L. Guipei, Y. Jianhai, Z.Peng, US20120009223 A1.

Unione Nazionale Industria Conciaria. Report Ambientale. Retrieved from: http://www.unic.it/it/servizi_associativi.php?id=44.

V. Beghetto, L. Agostinis, R. Taffarello and R. Samiolo, 2016. Innovative Active Cross-Linking Agents for Sustainable Leather Manufacturing. Springer International Publishing Switzerland, p. 149-155.

T. Kageyama, T. Osaki, J. Enomoto, D. Myasnikova, T. Nittami, T. Hozumi, T. Ito, J. Fukuda, 2016. In Situ Cross-Linkable Gelatin-CMC Hydrogels Designed for Rapid Engineering of Perfusable Vasculatures Biomater. Sci. Eng., 2, 1059-1066.

X. Shen, J. L. Shamshina, P. Berton, G. Gurauc, R. D. Rogers, 2016. Hydrogels based on cellulose and chitin:fabrication, properties, and applications. Green Chem., 18, 53-75.

A. Muñoz-Bonilla, M. Fernandez-Garcia, 2012. Polymeric materials with anti-microbial activity. Progress in Polymer Science, 37, 281-339.

V. Beghetto, PCT/IB2015/059892. 